ITC $3 / 50$

Information Technology and Control

Vol. 50 / No. $3 / 2021$

pp. 588-600

DOI 10.5755/j01.itc.50.3.25905
Distributed Iterative Learning Formation Control for Nonholonomic Multiple Wheeled Mobile Robots with Channel Noise

Received 2020/04/23

Accepted after revision 2021/04/15

HOW TO CITE: Xuhui, B., Yanling, Y., Wei, Y., Jiahao, G., Xinghe, M. (2021). Distributed Iterative Learning Formation Control for Nonholonomic Multiple Wheeled Mobile Robots with Channel Noise. Information Technology and Control, 50(3), 588-600 https://doi. org/10.5755/j01.itc.50.3.25905

\title{
Distributed Iterative Learning Formation Control for Nonholonomic Multiple Wheeled Mobile Robots with Channel Noise
}

\author{
Bu Xuhui, Hou Rui, Yin Yanling, Yu Wei, Geng Jiahao, Ma Xinghe \\ School of Electrical Engineering \& Automation, Henan Polytechnic University, Jiaozuo, China. \\ e-mails: buxuhui@gmail.com; hou.r@foxmail.com; yinylhpu@163.com; yuwei5150@163.com; \\ gjh809441455@126.com; maxinghe@hpu.edu.com
}

Corresponding author: yinylhpu@163.com

In this paper, a distributed iterative learning formation control (DILFC) algorithm for the robust formation problem of multiple non-holonomic wheeled mobile robots (WMRs) in repetitive motion environment was proposed, and the convergence under channel noise and switching communication topology was analyzed. Firstly, the consensus tracking error is constructed. Next, the controller is designed based on the predicted and the current learning term between robots, and the switching topology is introduced into the formation algorithm in the form of piecewise function. By adoption of a graph dependent matrix norm to derive a simpler convergence condition. And the norm upper bound for the tracking stability of the system are obtained. The results show that although the channel noise accumulates in both the time domain and iteration domain, the validity of formation can be guaranteed by adjusting the sampling time of the system. To illustrate the effectiveness of the proposed scheme, numerical simulation results of a group of WMRs are presented.

KEYWORDS: multiple wheeled mobile robots, formation tracking, iterative learning control, channel noise, switching topology. 


\section{Introduction}

In recent years, WMR has been widely used in the logistics industry, military reconnaissance, agricultural transportation and other practical projects [24], so the control of WMR has been rapidly developed, such as sliding mode control [27], backstepping control [7], adaptive control [15], feedback control [19] and observer-based control [1], etc.

Due to the limited performance of a single WMR, it is difficult to complete a large project alone, so multiple WMRs cooperative control is more practical. More and more scholars have done a lot of research on the cooperative control of multiple WMRs, where formation control is one of the important research directions. Formation control is a coordinated movement that a group of similar WMRs formed and maintain a certain formation in the process of movement. Traditional formation research methods mainly include leader-following formation [3], behavior-based method [2], virtual structure method [11] and based on graph theory distributed formation method [23]. Shao et al. [21] proposed a three-layer hybrid control architecture for controlling groups of mobile robots, to achieve formation based on the leader-following scheme. Ou et al. [16] transformed the tracking error of the robot into two subsystems and designed a continuous distributed controller for each robot by analyzing the two subsystems respectively, the group of WMRs can track the reference trajectory was achieved. In WMR formation control, battery power and the processor's computing ability is limited, so how to design more efficient communication and control methods between the WMRs to become a challenging problem to be solved.

The distributed control scheme allows computing work to be divided among all WMRs in the formation, each WMR sharing information with its leader only via wireless network, so the design of distributed formation control scheme becomes an important research direction. In [10] and [18], the consensus information is used to construct the formation error and design the distributed formation algorithm. However, all of the above control approaches are asymptotic stable control along the time domain. Although stable formation can be achieved, the transient performance of the system is poor at the initial stage.

In addition, there is a class of tasks that are run repeatedly with finite time interval in some practical applications of WMR. Most of the existing formation control algorithms are asymptotically stable control in time domain, but the transient performance was not ideal. Iterative learning control (ILC) is an effective solution for single WMR tracking trajectory in repetitive environment [22], which can achieve the complete tracking in time domain. ILC has been widely used in the field of collaborative control $[6,13$, 14]. Liu and Jia [14] studied the formation problem for discrete-time multi-agent systems with unknown nonlinear dynamics, a distributed iterative learning method was proposed based on the local formation error data and the sufficient conditions for preserving formation was deduced. The accurate formation was guaranteed in the presence of initial formation errors and switching topology network. $\mathrm{Li}$ and $\mathrm{Li}$ [13] considered the consensus tracking problem for a class of nonlinear multi-agent systems, an adaptive ILC consensus algorithm was developed, where the convergence condition was given based on the Lyapunov stability theory. Bu et al. [6] provided a model-free adaptive iterative learning consensus tracking strategy by using the dynamical linearization technique along with the iteration domain, to settle the consensus tracking problem for nonlinear multiagent systems. However, the above collaborative and formation algorithms were designed on the foundation of multi-agent systems. Since WMR is a typical nonlinear system with non-holonomic constraints, these algorithms cannot be directly applied to WMRs formation, so it is necessary to study multiple WMRs distributed formation algorithms based on ILC.

In practice, the data transmission of wireless communication network between WMRs is not always correct, which makes it more difficult to design and analyze the convergence of the controller. To solve the problem of ILC with network constraints, a series of solutions have been proposed in $[4,5,8,9,12$, 20, 26]. Zhang and Li [26] introduced the Sigma Delta quantizer with limited communication data rate into the control system, a quantized iterative learning control was designed and the analysis of convergence utilized a decreasing learning gain. to solve the tracking problem of continuous-time multi-agent system in the case of quantization and packet losses. $\mathrm{Bu}$ et al. [5] studied the robustness of the ILC algorithm for 
nonlinear systems with random measurement disturbance and proposed an improved iterative learning methodology of gain reduction based on p-type ILC, which can guarantee that the tracking error of the nonlinear system in presence of measurement disturbances to zero.

However, unlike measurement noise and state disturbance, channel noise is introduced externally. Because of the different positions it produces, channel noise can be divided into sensor-controller (SC) noise and controller-actuator (CA) noise. The former arises when exchanging measurement data between the sensor and the controller, and involves itself in the learning process. The latter arises when exchanging input data between the controller and the actuator, and affects the convergence of output error directly. Huang and Fang [8] studied the channel noise in ILC, and it was found that the contributions of SC and CA noise to errors were affected by the learning gain. Huang et al. [9] proposed an adaptive learning gain ILC to improve the convergence performance of the linear system with channel noise. However, WMR as a typical nonlinear system, the above strategy cannot be directly expanded to multiple WMRs formation control with channel noise.

Based on the above analysis, this paper designs a distributed iterative learning formation control algorithm for formation problem of multiple WMRs, and explores the convergence problem of the system under the channel noise and switching topologies. Compared with [26] and [5], this paper mainly analyzes the formation system of multiple mobile robots, which is more complex than multi-agent system. Compared with [9], this paper solves the convergence problem of channel noise in the nonlinear system of mobile robots and extends it to formation motion. The main contributions of this paper are as follows:

1 Compared with the traditional multiple WMRs formation algorithm, the proposed algorithm is based on the iteration axis instead of the time axis. The transient performance is better because it can track each time point in a limited running interval.

2 Based on the nonlinear system model of mobile robot, the consensus error is constructed. The current and predicted errors are introduced into the controller as learning items to improve the formation effect of the system under channel noise. The upper bound of formation error is obtained and the effect of channel noise on the formation tracking effect of multiple WMRs is analyzed.

3 The formation tracking algorithm of multiple WMRs under fixed topology is extended to the iteration-varying communication topology. By adoption of a graph dependent matrix norm, the convergence condition with gain is specified. Such a norm enables us to derive a simpler convergence condition.

The rest of this paper is organized as follows. In section 2 , the necessary preliminary work and the control objective are given respectively. In section 3 , the formation tracking controller is designed, and the influence of channel noise on formation tracking is analyzed. The numerical simulation is given in section 4 . Finally, we conclude this paper in section 5 .

\section{Preliminaries and Problem Formulation}

\subsection{Preliminaries}

In this paper, $R$ represents the set of real numbers, \| $A \|$ represents a matrix norm for the matrix $A \in R^{n \times n}$, $\operatorname{diag}\{\}$ represents the diagonal matrix and $I$ represents the identity matrix. On algebraic graph theory, the communication topology between WMRs can be described by a mathematical matrix. The weighted directed graph is denoted as $G=\{V, E, A\}$, where $V=\{1,2, \ldots, N\}$ is the set of vertexes, it represents the set of robots in this paper. $E \subseteq V \times V$ is the set of edges. $N_{i}=\{j \in V \mid(j, i) \in E\}$ denotes the neighborhood of robot i. $A \in R^{n \times n}$ express the weighted adjacency matrix of $G$, where $a_{i j}>0$, if $(i, j) \in E$, otherwise $a_{i j}=0$. The Laplacian matrix of $G$ is expressed by $L=D-A$, where $D=\operatorname{diag}\left\{d_{1}, d_{2}, \ldots, d_{n}\right\}$ is called the in-degree matrix and $d_{i}=\sum_{j=1}^{n} a_{i j}$. A directed graph is said to be strongly connected if a path exists between any two vertexes in the graph. Assume that the directed graph contains at least one spanning tree, with node 0 as the root.

\subsection{Problem Formulation}

We consider a group of $N$ WMRs, each robot has the same mechanical structure as shown in Fig. 1. $x_{i, k}(t), y_{i, k}(t), \theta_{i, k}(t)$ are the generalized position and 
Figure 1

Configuration of WMR

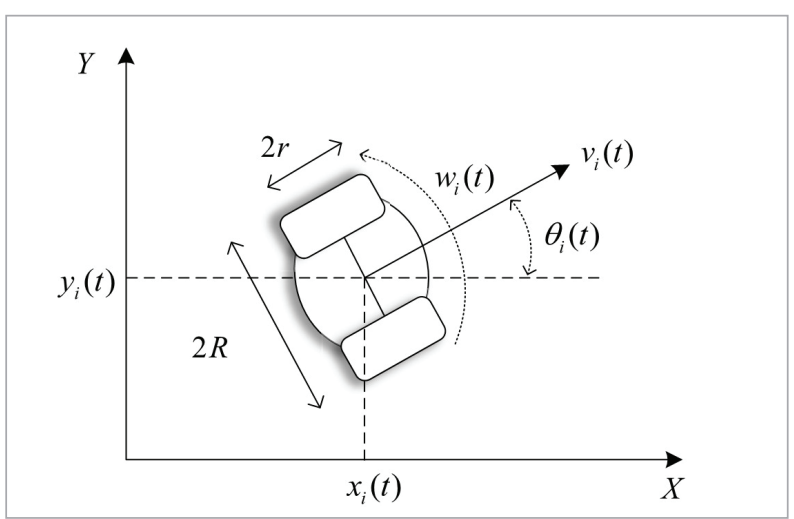

orientation of the WMR $i, i=1,2, \ldots N$. It is assumed that the wheels of the robot and the ground cannot move in a direct sideslip, then WMR has the following non-holonomic constraint,

$$
\begin{aligned}
& \frac{x_{i, k}(t+1)-x_{i, k}(t)}{\Delta T} \sin \theta_{i, k}(t)= \\
& \frac{y_{i, k}(t+1)-y_{i, k}(t)}{\Delta T} \cos \theta_{i, k}(t) .
\end{aligned}
$$

Under the non-holonomic constraint, the discrete-time model of the WMR $i$ at the k-th iteration in the form as follows:

$$
\left\{\begin{array}{l}
x_{i, k}(t+1)=x_{i, k}(t)+\Delta T \cos \theta_{i, k}(t) v_{i, k}(t) \\
y_{i, k}(t+1)=y_{i, k}(t)+\Delta T \sin \left(\theta_{i, k}(t)\right) v_{i, k}(t), \\
\theta_{i, k}(t+1)=\theta_{i, k}(t)+\Delta T w_{i, k}(t)
\end{array}\right.
$$

where $t \in[0, T]$ is the discrete time, $k=1,2, \ldots$ is iteration times, $\Delta T$ is the sampling time. $v_{i, k}(t), w_{i, k}(t)$ are linear and angular velocities respectively.

The states matrix, velocities matrix and control input matrix of WMR $i$ are represented by $\eta_{i, k}(t), u_{i, k}(t), B\left(\eta_{i, k}(t)\right)$ respectively,

$$
\begin{aligned}
& \eta_{i, k}(t)=\left[\begin{array}{lll}
x_{i, k}(t) & y_{i, k}(t) & \theta_{i, k}(t)
\end{array}\right]^{T}, \\
& u_{i, k}(t)=\left[\begin{array}{lll}
v_{i, k}(t) & w_{i, k}(t)
\end{array}\right]^{T}, \\
& B\left(\eta_{i, k}(t)\right)=\Delta T\left[\begin{array}{ll}
\cos \theta_{i, k}(t) & 0 \\
\sin \theta_{i, k}(t) & 0 \\
0 & 1
\end{array}\right] .
\end{aligned}
$$

Then equation (2) can be rewritten as follows:

$$
\eta_{i, k}(t+1)=\eta_{i, k}(t)+B\left(\eta_{i, k}(t)\right) u_{i, k}(t) .
$$

The non-holonomic WMR model (3) adopted in this paper satisfies the following properties:

Property 1: The nonlinear matrix function $B\left(\eta_{i, k}(t)\right)$ is globally Lipschitz in $\eta_{i, k}(t)$, that is

$\left\|B\left(\eta_{i, k}(t)\right)-B_{i}\left(\eta_{\mathrm{j}, k}(t)\right)\right\| \leq l_{b}\left\|\eta_{i, k}(t)-\eta_{\mathrm{j}, k}(t)\right\|$,

for $\forall t \in[0, T]$, and $l_{b}$ is a positive constant.

Property 2: the matrix function $B\left(\eta_{i, k}(t)\right)$ is bound as $\left\|B\left(\eta_{i, k}(t)\right)\right\| \leq b_{B}$, where $b_{B}$ is a positive constant.

In this paper, the reference trajectory $\eta_{d}$ is regarded as the running track of a virtual leader WMR, and the virtual leader is denoted as number 0 . Defining $S=\operatorname{diag}\left\{s_{1}, \ldots, s_{j}, \ldots, s_{n}\right\}$, where $s_{i}=1$ if the WMR $i$ can obtain the reference trajectory, otherwise $s_{i}=0$. We assume that the reference trajectory $\eta_{d}(t+1)$ is known, there is a bounded expected control input $u_{d}(t)$, satisfying

$\eta_{d}(t+1)=\eta_{d}(t)+B\left(\eta_{d}(t)\right) u_{d}(t)$,

where $\left\|u_{d}(t)\right\| \leq b_{u d}$.

The goal of formation control in this paper is designing a suitable control algorithm for the velocity $u_{k}(t)$ of WMR, so that all WMRs can maintain the desired formation during the tracking along the reference track. For all $t \in[0, T]$ and $i=1,2, \ldots N$, the following equation is satisfied:

$\lim _{k \rightarrow \infty} \eta_{i, k}(t)=\eta_{d}(t)+d_{i}(t)$,

where $d_{i}(t)$ denotes the desired trajectory deviation of WMR $i$. Different WMR may have different desired trajectory, ie, $d_{i}(t) \neq d_{j}(t)$ for $i \neq j$. In addition, objective (5) implies $\lim _{k \rightarrow \infty} \eta_{j, k}(t)-\eta_{i, k}(t)=d_{j i}(t)$.

According to the rules of communication transmission between adjacent WMRs, the consensus error is given:

$$
\begin{aligned}
\xi_{i, k}(t)= & \sum_{j \in N_{i}} a_{i, j}\left(\eta_{j, k}(t)-\eta_{i, k}(t)+d_{i j}(t)\right) \\
& +s_{i}\left(\eta_{d}(t)-\eta_{i, k}(t)+d_{i}(t)\right) .
\end{aligned}
$$

where $a_{i, j} \in A$ express the communication of the WMR $i$ and $j$ in the adjacency matrix. 
Let $\delta \eta_{i, k}(t)=\eta_{d}(t)+d_{i}(t)-\eta_{i, k}(t)$ express the tracking error of the WMR $i$.

When WMRs transmit information over the wireless network, the input $u_{i, k}(t)$ and output error $\delta \eta_{i, k}(t)$ need to be transmitted from the controller to the actuator and form the sensor to the controller respectively. The network model is shown in Fig. 2.

\section{Figure 2}

The WMR system transmits information over wireless networks

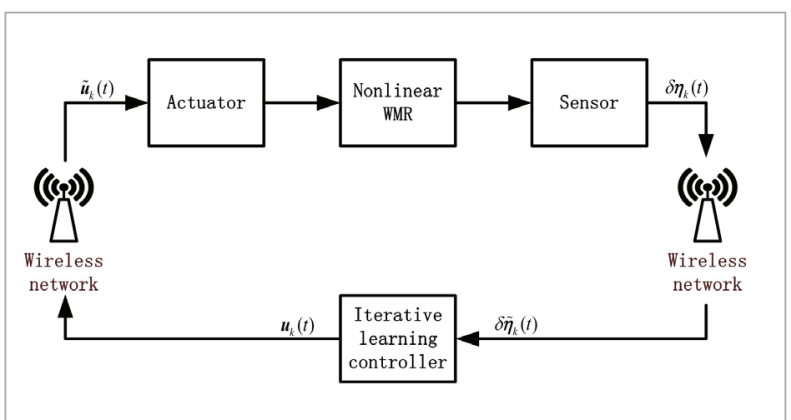

The CA and SC channel noise are represented by $m(t), n(t)$ respectively, then the tracking error and the input data can be represented as

$$
\begin{aligned}
& \tilde{u}_{k}(t)=u_{k}(t)+m_{k}(t), \\
& \delta \tilde{\eta}_{k}(t)=\delta \eta_{k}(t)+n_{k}(t) .
\end{aligned}
$$

then the consensus error can be rewritten as

$$
\tilde{\xi}_{i, k}(t)=\sum_{j \in N_{i}} a_{i, j}\left(\delta \tilde{\eta}_{i, k}(t)-\delta \tilde{\eta}_{j, k}(t)\right)+s_{i}\left(\delta \tilde{\eta}_{i, k}(t)\right) .
$$

Remark 1: Due to the unreliability of the wireless network, the output errors $\delta \eta_{i, k}(t)$ received by the controller and input data $u_{i, k}(t)$ obtained by the actuator will be mixed with the channel noise during the transmission.

\section{Control Algorithm Design and Convergence Analysis}

To design a formation algorithm under iteration-varying communication topology, we design a switching signal function $\sigma(k):[0, \infty) \mapsto\{1, \ldots, \mathrm{M}\}$ to describe variable communication topology, where $s \in\{1, \ldots, \mathrm{M}\}$ and $G_{\sigma}=\left\{G_{1}, \ldots, G_{M}\right\}$ are identical in quantity and order. In the case that the topology structure changes with iteration, the model and consensus formation error are shown as follows

$$
\begin{gathered}
\eta_{i, k}{ }^{*}(t+1)=\eta_{i, k}(t)+B\left(\eta_{i, k}(t)\right) \tilde{u}_{i, k}(t), \\
\xi_{i, k}{ }^{*}(t)=\sum_{j \in N_{i}} a_{i j, k}(t)\left(\delta \tilde{\eta}_{i, k}(t)-\delta \tilde{\eta}_{j, k}(t)\right) \\
+s_{i}(t)\left(\delta \tilde{\eta}_{i, k}(t)\right) .
\end{gathered}
$$

With the above consensus error, the DILFC algorithm as shown in Fig. 3 is proposed:

$u_{i, k+1}(t)=u_{i, k}(t)+\Gamma_{i, 1}(t) \xi_{i, k}{ }^{*}(t+1)+\Gamma_{i, 2}(t) \xi_{i, k+1}{ }^{*}(t),(12)$

where $\Gamma_{1}(t)$ and $\Gamma_{2}(t)$ are bounded learning gain matrices. The control signal consists is composed of the predicted learning term $\Gamma_{1}(t) \xi_{i, k}{ }^{*}(t+1)$ at time $t+1$ that from the kth iteration and the current term $\Gamma_{1}(t) \xi_{i, k+1}{ }^{*}(t)$ can improve the stability of the system under the unpredictable uncertainty.

\section{Figure 3}

Block diagram of the scheme performed for WMR

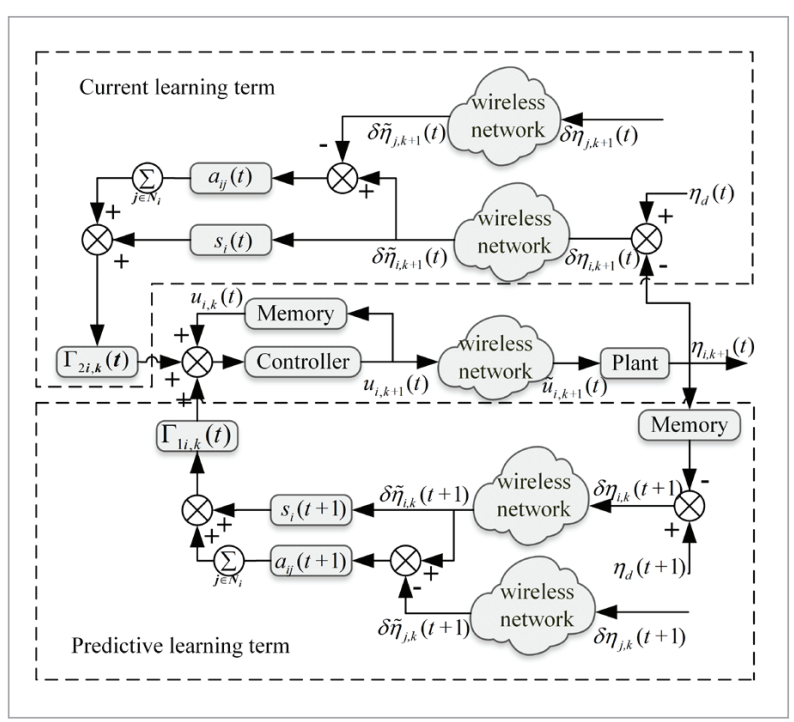

To facilitate the analysis, we have the following assumptions:

Assumption 1: The initial state of each WMR is the same in every iteration and $x_{i, k}(0)=x_{d}(0)$. 
Assumption 2: The channel noise $m(t), n(t)$ are bounded for every iteration, that is $\max _{0 \leq t \leq T}\left\|m_{k}(t)\right\| \leq b_{m}$, $\max _{0 \leq T}\left\|n_{k}(t)\right\| \leq b_{n}$, where directional $b_{m}, b_{n}$ are positive constant.

Assumption 3: Each switching communication graph has a spanning tree with a virtual leader as the root.

Remark 2. Assumption 1 is a basic condition of the ILC system, the identical initial condition is still followed in this paper. Furthermore, in [9], channel noise is assumed to be bounded, and the same assumption is still adopted in this paper. So, Assumption 1 and Assumption 2 are reasonable.

Definition: The $\alpha$-norm is defined for a function $\boldsymbol{h}:[0, T] \rightarrow \mathbf{R}^{n}$ as

$$
\|\boldsymbol{h}(\cdot)\|_{\alpha}=\sup _{t \in T} \boldsymbol{h}(t)\left(\frac{1}{\alpha}\right)^{t}, \alpha>1 .
$$

For convenience, we define $\delta_{i}, i=1,2, \ldots, N$ as the $i$ th eigenvalue of $L+S$. Based on these assumptions, the following theorem gives the main results of this paper.

Theorem: Considering multiple WMRs satisfies assumptions 1 3, the DILFC controller is adopted shown in equation (12). If the gain matrix satisfies

$\max _{i=1,2 . N} \max _{t \in[0, T]} \rho_{1}\left(I-\delta_{i} \Gamma_{1} B\left(\eta_{i, k}(t)\right)\right) \leq \sigma<1$.

The $\alpha$-norm of the system consensus error is bounded, and its upper bound is

$$
\begin{aligned}
& \lim _{k \rightarrow \infty}\left\|\eta_{i, k}(t)-\eta_{j, k}(t)\right\|_{\alpha} \\
& \leq 2 b_{B}\left(\frac{1-\left(\frac{s}{\alpha}\right)^{T}}{\alpha-s}\right) \cdot \frac{\varepsilon}{1-\bar{\rho}(t)}+2 b_{B} b_{m}\left(\frac{1-\left(\frac{s}{\alpha}\right)^{T}}{\alpha-s}\right) .
\end{aligned}
$$

Proof: To simplify the analysis, the compact form of $\eta_{i, k}(t), u_{i, k}(t), \delta \eta_{i, k}(t)$ and the channel noise are given

$$
\begin{aligned}
& \boldsymbol{\eta}_{k}(t)=\left[\eta_{1, k}(t)^{T}, \eta_{2, k}(t)^{T}, \ldots, \eta_{N, k}(t)^{T}\right]^{T}, \\
& \boldsymbol{u}_{k}(t)=\left[u_{1, k}(t)^{T}, u_{2, k}(t)^{T}, \ldots, u_{N, k}(t)^{T}\right]^{T}, \\
& \boldsymbol{n}_{k}(t)=\left[n_{1, k}(t)^{T}, n_{2, k}(t)^{T}, \ldots, n_{N, k}(t)^{T}\right]^{T} \\
& \boldsymbol{m}_{k}(t)=\left[m_{1, k}(t)^{T}, m_{2, k}(t)^{T}, \ldots, m_{N, k}(t)^{T}\right],^{T} \\
& \delta \boldsymbol{\eta}_{k}(t)=\left[\delta \eta_{1, k}(t)^{T}, \delta \eta_{2, k}(t)^{T}, \ldots, \delta \eta_{N, k}(t)^{T}\right] .{ }^{T}
\end{aligned}
$$

The multiple WMRs model can be written as

$$
\boldsymbol{\eta}_{k}(t+1)=\boldsymbol{\eta}_{k}(t)+B\left(\boldsymbol{\eta}_{k}(t)\right) \boldsymbol{u}_{k}(t) .
$$

Similarly, a compact form of consensus error can be given

$$
\xi_{k}(t)=((L+S) \otimes I) \delta \eta_{k}(t),
$$

where

$\boldsymbol{\xi}_{k}(t)^{T}=\left(\xi_{1, k}(t)^{T}, \xi_{2, k}(t)^{T}, \ldots, \boldsymbol{\xi}_{N, k}(t)^{T}\right)^{T}, \xi_{i, k}(t) \in \boldsymbol{R}^{n}$.

Then the system state and consensus error under channel noise are rewritten as

$$
\boldsymbol{\eta}_{k}^{*}(t+1)=\boldsymbol{\eta}_{k}(t)+B\left(\boldsymbol{\eta}_{k}(t)\right) \tilde{\boldsymbol{u}}_{k}(t),
$$

$$
\xi_{k}^{*}(t)=((L+S) \otimes I) \delta \tilde{\eta}_{k}(t) .
$$

Let $H_{k}=L+S$, where $k$ represents change with iteration, substitute (18) into (12)

$$
\begin{aligned}
\boldsymbol{u}_{k+1}(t) & =\boldsymbol{u}_{k}(t)+\left(I_{N} \otimes \Gamma_{1}(t)\right) \boldsymbol{\xi}_{k}^{*}(t+1) \\
& +\left(I_{N} \otimes \Gamma_{2}(t)\right) \boldsymbol{\xi}_{k+1}{ }^{*}(t) \\
& =\boldsymbol{u}_{k}(t)+\left(H_{k} \otimes \Gamma_{1}(t)\right) \delta \tilde{\boldsymbol{\eta}}_{k}(t+1) \\
& +\left(H_{k} \otimes \Gamma_{2}(t)\right) \delta \tilde{\boldsymbol{\eta}}_{k+1}(t+1) .
\end{aligned}
$$

According to (17) and (18), the input error could be expressed as

$$
\begin{aligned}
& \delta \boldsymbol{u}_{k+1}(t)=\boldsymbol{u}_{d}(t)-\boldsymbol{u}_{k+1}(t) \\
& =\boldsymbol{u}_{d}(t)-\boldsymbol{u}_{k}(t)-\Gamma_{1}(t) \boldsymbol{\xi}_{k}^{*}(t+1)-\Gamma_{2}(t) \boldsymbol{\xi}_{k+1}^{*}(t) \\
& =\left[I-H_{k} \otimes \Gamma_{1}(t) B\left(\boldsymbol{\eta}_{k}(t)\right)\right] \delta \boldsymbol{u}_{k}(t)-H_{k} \\
& \otimes \Gamma_{1}(t) \delta \boldsymbol{\eta}_{k}(t)-H_{k} \otimes \Gamma_{1}(t)\left(B \left(\eta_{d}(t)-B\left(\boldsymbol{\eta}_{k}(t)\right) \boldsymbol{u}_{d}(t)\right.\right. \\
& -H_{k} \otimes \Gamma_{1}(t) B\left(\boldsymbol{\eta}_{k}(t)\right) \boldsymbol{m}_{k}(t+1)-H_{k} \otimes \Gamma_{1}(t) \\
& \times \boldsymbol{n}_{k}(t+1)-H_{k} \otimes \Gamma_{2}(t) \delta \boldsymbol{\eta}_{k+1}(t)-H_{k} \otimes \Gamma_{2}(t) \boldsymbol{n}_{k+1}(t) .
\end{aligned}
$$

Taking norm on both sides of (16), we can get

$$
\begin{gathered}
\left\|\delta \boldsymbol{u}_{i, k+1}(t)\right\| \leq \rho(t)\left\|\delta \boldsymbol{u}_{i, k}(t)\right\|+l_{1}\left\|\delta \boldsymbol{\eta}_{i, k}(t)\right\| \\
+l_{2}\left\|\delta \boldsymbol{\eta}_{i, k+1}(t)\right\|+l_{3},
\end{gathered}
$$

where

$\rho(t)=\left\|I-H_{k} \otimes \Gamma_{1}(t) B\left(\boldsymbol{\eta}_{k}(t)\right)\right\|$,

$l_{1}=\left\|H_{k} \otimes \Gamma_{1}(t)\right\|\left(1+b_{u d}\right)$,

$l_{2}=\left\|H_{k} \otimes \Gamma_{2}(t)\right\|$,

$l_{3}=\left\|H_{k} \otimes \Gamma_{1}(t)\right\|\left(b_{B} b_{m}+b_{n}\right)+\left\|H_{k} \otimes \Gamma_{2}(t)\right\| b_{n}$. 
Form and the input error could be expressed as

$$
\begin{aligned}
& \delta \boldsymbol{\eta}_{k}(t+1)=\boldsymbol{\eta}_{d}(t+1)-\boldsymbol{\eta}_{k}^{*}(t+1) \\
& \quad=\boldsymbol{\eta}_{d}(t)+\boldsymbol{B}\left(\boldsymbol{\eta}_{d}(t)\right) \boldsymbol{u}_{d}(t)-\boldsymbol{\eta}_{k}(t)-\boldsymbol{B}\left(\boldsymbol{\eta}_{k}(t)\right) \tilde{\boldsymbol{u}}_{k}(t) \\
& =\delta \boldsymbol{\eta}_{k}(t)+\left(\boldsymbol{B}\left(\boldsymbol{\eta}_{d}(t)\right)-\boldsymbol{B}\left(\boldsymbol{\eta}_{k}(t)\right)\right) \boldsymbol{u}_{d}(t) \\
& +\boldsymbol{B}\left(\boldsymbol{\eta}_{k}(t)\right) \delta \tilde{\boldsymbol{u}}_{k}(t) .
\end{aligned}
$$

Take the norm of both sides of the above equation and consider Property 1, Property 2 and Assumption 2, it can be seen that

$$
\begin{aligned}
& \left\|\delta \boldsymbol{\eta}_{k}(t+1)\right\| \leq\left\|\delta \boldsymbol{\eta}_{k}(t)\right\|+l_{b}\left\|\delta \boldsymbol{\eta}_{k}(t)\right\|\left\|u_{d}(t)\right\| \\
& \quad+\left\|\boldsymbol{B}\left(\boldsymbol{\eta}_{k}(t)\right)\right\|\left\|\delta \tilde{\boldsymbol{u}}_{k}(t)\right\| \\
& \quad \leq\left(1+l_{b} b_{u d}\right)\left\|\delta \boldsymbol{\eta}_{k}(t)\right\|+b_{B}\left\|\delta \tilde{\boldsymbol{u}}_{k}(t)\right\| \\
& \quad \leq z\left\|\delta \boldsymbol{\eta}_{k}(t)\right\|+b_{B}\left\|\delta \tilde{\boldsymbol{u}}_{k}(t)\right\|,
\end{aligned}
$$

where $z=\left(1+l_{b} b_{u d}\right)$.

Based on Assumption 1, (23) can be rewritten as

$$
\begin{aligned}
& \left\|\delta \boldsymbol{\eta}_{k}(t)\right\| \leq \sum_{h=0}^{t-1} z^{t-1-h} b_{B}\left\|\delta \tilde{\boldsymbol{u}}_{k}(h)\right\|+q^{t}\left\|\delta \boldsymbol{\eta}_{k}(0)\right\| \\
& \leq \sum_{h=0}^{t-1} z^{t-1-h} b_{B}\left(\| \delta \boldsymbol{u}_{k}(h)+b_{m}\right) .
\end{aligned}
$$

Substituting (24) into (21), it can be seen that

$$
\begin{aligned}
\left\|\delta \boldsymbol{u}_{k+1}(t)\right\| & \leq \rho(t)\left\|\delta \boldsymbol{u}_{k}(t)\right\| \\
& +l_{1} \sum_{h=0}^{t-1} z^{t-1-h} b_{B}\left(\left\|\delta \boldsymbol{u}_{k}(h)\right\|+b_{m}\right) \\
& +l_{2} \sum_{h=0}^{t-1} z^{t-1-h} b_{B}\left(\left\|\delta \boldsymbol{u}_{k+1}(h)\right\|+b_{m}\right)+l_{3} .
\end{aligned}
$$

Multiplying both sides of (25) by $(1 / \alpha)^{t}$ to compute $\alpha$-norm of the input error, where $\alpha>\max [1, z]$, we can have

$$
\begin{aligned}
& \left\|\delta \boldsymbol{u}_{k+1}(t)\right\|\left(\frac{1}{\alpha}\right)^{t} \leq \rho(t)\left\|\delta \boldsymbol{u}_{k}(t)\right\|\left(\frac{1}{\alpha}\right)^{t}+\left(\frac{l_{1}}{\alpha}\right)^{t} \\
& \times \sum_{h=0}^{t-1}\left(\frac{z}{\alpha}\right)^{t-1-h} b_{B}\left(\left\|\delta \boldsymbol{u}_{k}(h)\right\|\left(\frac{1}{\alpha}\right)^{h}+b_{m}\left(\frac{1}{\alpha}\right)^{h}\right)+\left(\frac{l_{2}}{\alpha}\right)^{t} \\
& \times \sum_{h=0}^{t-1}\left(\frac{z}{\alpha}\right)^{t-1-h} b_{B}\left(\left\|\delta \boldsymbol{u}_{k+1}(h)\right\|\left(\frac{1}{\alpha}\right)^{h}+b_{m}\left(\frac{1}{\alpha}\right)^{h}\right)+l_{3}\left(\frac{1}{\alpha}\right)^{t}
\end{aligned}
$$

Since the norm of a constant is still a constant, (26) can be written as follows

$$
\begin{aligned}
& \left\|\delta \boldsymbol{u}_{k+1}(t)\right\|_{\alpha} \\
& \leq b_{B}\left(\left\|\delta \boldsymbol{u}_{k+1}(t)\right\|_{\alpha}+b_{m}\right)\left(\frac{l_{2}}{\alpha}\right) \sum_{h=0}^{t-1}\left(\frac{z}{\alpha}\right)^{t-1-h}+\rho(t)\left\|\delta \boldsymbol{u}_{k}(t)\right\|_{\alpha} \\
& +b_{B}\left(\left\|\delta \boldsymbol{u}_{k}(h)\right\|_{\alpha}+b_{m}\right)\left(\frac{l_{1}}{\alpha}\right) \sum_{h=0}^{t-1}\left(\frac{z}{\alpha}\right)^{t-1-h}+l_{3} \\
& \leq\left(\rho(t)+b_{B} l_{1}\left(\frac{1-(z / \alpha)^{T}}{\alpha-z}\right)\right)\left\|\delta \boldsymbol{u}_{k}(t)\right\|_{\alpha}+ \\
& b_{B} l_{2}\left(\frac{1-(z / \alpha)^{T}}{\alpha-z}\right)\left\|\delta \boldsymbol{u}_{k+1}(t)\right\|_{\alpha}+2 b_{B} l_{1} b_{m}\left(\frac{1-(z / \alpha)^{T}}{\alpha-z}\right)+l_{3} \\
& \leq \bar{\rho}(t)\left\|\delta \boldsymbol{u}_{k}(t)\right\|_{\alpha}+\varepsilon
\end{aligned}
$$

where

$$
\bar{\rho}(t)=\frac{\rho(t)+b_{B} l_{1}\left(\frac{1-(z / \alpha)^{T}}{\alpha-z}\right)}{1-b_{B} l_{2}\left(\frac{1-(z / \alpha)^{T}}{\alpha-z}\right)}, \varepsilon=\frac{2 b_{B} l_{1} b_{m}\left(\frac{1-(z / \alpha)^{T}}{\alpha-z}\right)+l_{3}}{1-b_{B} l_{2}\left(\frac{1-(z / \alpha)^{T}}{\alpha-z}\right)} .
$$

Because

$$
\begin{aligned}
& \left(\frac{l_{1}}{\alpha}\right) \sum_{h=0}^{t-1}\left(\frac{z}{\alpha}\right)^{t-1-h}=\left(\frac{l_{1}}{\alpha}\right)\left(\frac{z}{\alpha}\right)^{t-1} \sum_{h=0}^{t-1}\left(\frac{z}{\alpha}\right)^{-h} \\
& =\left(\frac{l_{1}}{\alpha}\right)\left(\frac{z}{\alpha}\right)^{t-1} \sum_{h=0}^{t-1}\left(\frac{\alpha}{z}\right)^{h}=\left(\frac{1}{\alpha}\right)\left(\frac{z}{\alpha}\right)^{t-1} \frac{1-((\alpha / z))^{t}}{1-(\alpha / z)} \\
& =\frac{(z / \alpha)^{t-1}\left((\alpha / z)^{t}-1\right)}{\alpha((\alpha / z)-1)}=\frac{1-(z / \alpha)^{t}}{\alpha-z} \leq \frac{1-(z / \alpha)^{T}}{\alpha-z} .
\end{aligned}
$$

Then, the (27) equals

$$
\begin{gathered}
\left\|\delta \boldsymbol{u}_{k+1}(t)\right\|_{\alpha} \leq \bar{\rho}^{k+1}(t)\left\|\delta \boldsymbol{u}_{0}(t)\right\|_{\alpha}+\varepsilon \sum_{h=0}^{k} \bar{\rho}^{h}(h) \\
=\bar{\rho}^{k+1}(t)\left\|\delta \boldsymbol{u}_{0}(t)\right\|_{\alpha}+\frac{\varepsilon\left(1-\bar{\rho}^{k+1}(t)\right)}{1-\bar{\rho}(t)} .
\end{gathered}
$$

Define $M \triangleq I-H_{k} \otimes \Gamma_{1}(t) B\left(\boldsymbol{\eta}_{k}(t)\right)$. There is always a matrix norm ensure $\rho(t)=\|M\| \leq \sigma<1$ when $\rho_{1}(M) \leq \sigma<1$. Further, we can find $\alpha$ large enough so that $\bar{\rho}(t)<1$. Then the upper bound of input error of mobile robot $i$ can be expressed as

$$
\lim _{k \rightarrow \infty}\left\|\delta \boldsymbol{u}_{k}(t)\right\|_{\alpha} \leq \frac{\varepsilon}{1-\bar{\rho}(t)} .
$$

Through the above analysis, it is shown that the input converges gradually after multiple learning and iterations. To investigate the task target of formation tracking, this paper makes the following treatment: 
Similarly, multiplying both sides of (24) by (1/ $\alpha)^{t}$ to compute $\alpha$-norm of the state error, we get

$$
\begin{aligned}
& \left\|\delta \boldsymbol{\eta}_{k}(t)\right\|_{\alpha} \\
& \quad \leq \frac{1}{\alpha} \sum_{h=0}^{t-1}\left(\frac{z}{\alpha}\right)^{t-1-h} b_{B}\left(\left\|\delta \boldsymbol{u}_{k}(t)\right\|\left(\frac{1}{\alpha}\right)^{h}+b_{m}\left(\frac{1}{\alpha}\right)^{h}\right) \\
& \quad \leq b_{B}\left(\left\|\delta \boldsymbol{u}_{k}(t)\right\|_{\alpha}+b_{m}\right)\left(\frac{1-(z / \alpha)^{T}}{\alpha-z}\right) \\
& \quad \leq b_{B}\left(\frac{\varepsilon}{1-\bar{\rho}(t)}+b_{m}\right)\left(\frac{1-(z / \alpha)^{T}}{\alpha-z}\right) .
\end{aligned}
$$

According to the above results, the formation error of the system is related to the channel noise $\boldsymbol{m}_{k}(t)$ and $\boldsymbol{n}_{k}(t)$. Hence, when the channel noise is 0 , $\lim _{k \rightarrow \infty}\left\|\delta \eta_{k}(t)\right\|_{\alpha}=0$.

Note that $M \in R^{3 N \times 3 N}$ is a complex matrix, and the condition $\rho_{1}(M) \leq \sigma<1, k \in[0, T]$ is specified since it includes all WMRs' kinematics and the complete communication topology. Next, we derive the convergence condition. For simplicity in the sequel, the time parameter is removed when no confusion arises.

A matrix norm operation [25] is defined:

$$
|\cdot| \triangleq\left|\left[\left(Q U^{*}\right) \otimes I\right](\cdot)\left[\left(U Q^{-1}\right) \otimes I\right]\right|,
$$

where $U$ is a unitary matrix satisfying $\Lambda=$ $U^{*}(L+S) U . \Lambda$ is an upper triangular matrix with diagonal entries being the eigenvalues of $L+S$, and ${ }^{*}$ denotes matrix conjugate transpose. $Q=\operatorname{diag}\left\{\beta, \beta^{2}, \ldots \beta^{N}\right\}, \beta \neq 0$ is a constant matrix. Then, we can do the following calculation,

$$
\begin{aligned}
& |M| \\
& \triangleq \mid\left[\left(Q U^{*}\right) \otimes I\right]\left[I-H_{k} \otimes \Gamma_{1}(t) B\left(\eta_{k}(t)\right)\right] \\
& \times\left[\left(U Q^{-1}\right) \otimes I\right] \\
& =\left|I-\left[\left(Q U^{*}\right) H_{k}\left(U Q^{-1}\right)\right] \otimes \Gamma_{1}(t) B\left(\eta_{k}(t)\right)\right| \\
& =\left|I-\left[\left(Q \Lambda Q^{-1}\right)\right] \otimes \Gamma_{1}(t) B\left(\eta_{k}(t)\right)\right| \\
& =\mid \begin{array}{c}
I-\delta_{1} \Gamma_{1}(t) B\left(\eta_{1, k}(t)\right) \\
0 \\
\vdots \\
0 \\
(*)
\end{array} \\
& \text { (*) } \\
& \ddots \\
& \left.0 \quad I-\delta_{N} \Gamma_{N}(t) B\left(\eta_{N, k}(t)\right)\right]
\end{aligned}
$$

By the Assumption3, then, all the eigenvalues of $H_{k}$ have positive real parts. Thus, we can conclude that if

$$
\max _{i=1,2 . . N} \max _{t \in[0, \mathrm{~T}]} \rho_{1}\left(I-\delta_{i} \Gamma_{1}(t) B\left(\eta_{i, k}(t)\right)\right) \leq \sigma<1 .
$$

Then $\left\|I-H_{K} \otimes \Gamma_{1}(t) B\left(\eta_{i, k}(t)\right)\right\| \leq \sigma<1$ holds.

This completes the proof.

By analyzing the above results, it can be found that the SC and CA channel noise will be accumulated along the iteration axis and time axis simultaneously. However, the summation of $\boldsymbol{m}_{k}(t)$ and $\boldsymbol{n}_{k}(t)$ is limited by the upper bound of nonlinear function $\boldsymbol{B}\left(\boldsymbol{\eta}_{k}(t)\right)$, because $\boldsymbol{B}\left(\boldsymbol{\eta}_{k}(t)\right)$ is composed of sampling time $\Delta T$, and the value of $\Delta T$ is far less than 1 . Therefore, the accumulated channel noise in the iterative domain and time domain can be significantly suppressed by the sampling time.

Remark 3: The formation control of a class of robots with repetitive motion is studied in this paper. In some practical applications, such as the mobile robot of logistics warehouse performing the throwing task and the agricultural robot performing the farm work task, which have strong repeatability. The analysis and design method in this paper can be directly extended.

\section{Simulation}

In this section, the formation tracking simulation of four WMRs by wireless network control is provided, and the communication topology changing with iteration is adopted. To verify the research effect of the previous section, we compared the influence of channel noise on the formation at different sampling times.

We consider the following reference trajectory:

$\eta_{d}(t)=\left\{\begin{array}{c}x_{d}(t)=\cos ((k-1) \times \pi \times \Delta T) \\ y_{d}(t)=\sin ((k-1) \times \pi \times \Delta T) \\ \theta_{d}(t)=\Delta T \times \pi \times(k-1)+\pi / 2\end{array} \quad, k=[0,2 \pi]\right.$.

The desired trajectory deviation

$$
\begin{aligned}
& d_{1}=\left[\begin{array}{c}
0 \\
-0.04
\end{array}\right], d_{2}=\left[\begin{array}{c}
0 \\
-0.08
\end{array}\right], \\
& d_{3}=\left[\begin{array}{l}
-0.08 \\
-0.16
\end{array}\right], d_{4}=\left[\begin{array}{c}
0.08 \\
-0.16
\end{array}\right] .
\end{aligned}
$$


In addition, the initial state of each WMR satisfies assumption 1, that is, the initial state of each iteration is the same as that of the expected trajectory, which is set the following:

$\eta_{1, k}(0)=[1,-0.04, \pi / 2]^{T}$,

$\eta_{2, k}(0)=[1,-0.08, \pi / 2]^{T}$,

$\eta_{3, k}(0)=[0.02,-0.16, \pi / 2]^{T}$,

$\eta_{4, k}(0)=[1.08,-0.16, \pi / 2]^{T}$.

And the control input is:

$v_{1, k}(0)=[0,0]^{T}, v_{2, k}(0)=[0,0]^{T}$,

$v_{3, k}(0)=[0,0]^{T}, v_{4, k}(0)=[0,0]^{T}$.

Here are three communication diagrams for switching between WMRs, this is shown Fig. 4. It can be observed that only WMR 1 can receive the information of the leader, assuming that the information transmission between WMRs is directional. Then $S=\operatorname{diag}(1,0,0,0)$, the Laplacian matrix can be known as:

$L_{1}=\left[\begin{array}{cccc}1 & -1 & 0 & 0 \\ -1 & 1 & 0 & 0 \\ 0 & -1 & 1 & 0 \\ -1 & 0 & 0 & 1\end{array}\right], L_{2}=\left[\begin{array}{cccc}1 & -1 & 0 & 0 \\ -1 & 1 & 0 & 0 \\ 0 & -1 & 1 & 0 \\ -1 & 0 & 0 & 1\end{array}\right]$,

$L_{3}=\left[\begin{array}{cccc}1 & -1 & 0 & 0 \\ -1 & 1 & 0 & 0 \\ -1 & 0 & 1 & 0 \\ 0 & -1 & 0 & 1\end{array}\right]$.

A randomly switching signal function $\sigma(k)$ is defined and the value 1,2,3 respectively represents the corresponding topology. Fig. 5 shown the switching signal $\sigma(k)$ in this simulation. The switching sequence takes the corresponding value as iteration changes. To ensure that the probability of each switching sig-

\section{Figure 4}

Communication topologies among WMRs

Communication topologies among WMRs

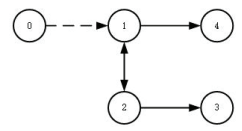

(a)

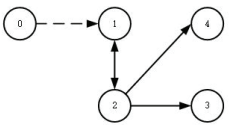

(b)

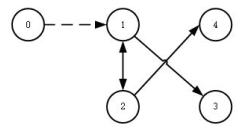

(c)
Figure 5

Switching signal among there different communication topologies

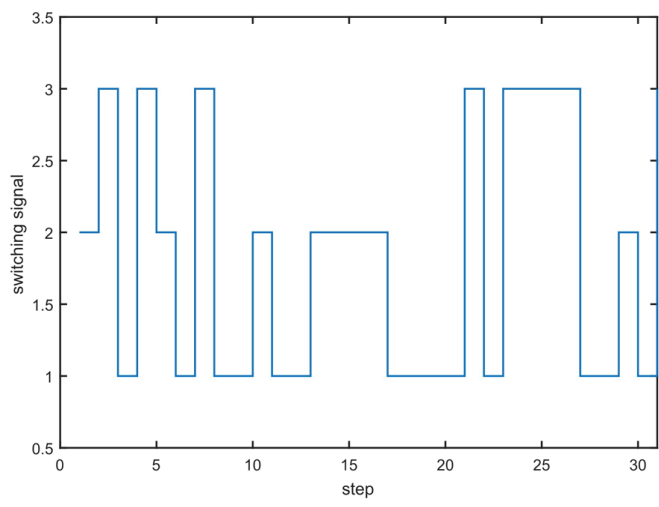

nal is equal, the rules for switching signal function $\sigma(k)$ as follows

1 If $\sigma(k) \in[0,0.33), s_{i}=1$;

$2 \sigma(k) \in[0.33,0.66), s_{i}=2$;

$3 \sigma(k) \in[0.66,1], s_{i}=3$.

The distributed algorithm (12) is adopted here, and the learning gain matrix $\Gamma_{1 i, k}(t)$ and $\Gamma_{2 i, k}(t)$ are chosen as

$$
\begin{aligned}
& \Gamma_{1 i, k}(t)=1.6\left[\begin{array}{ccc}
\cos \theta_{i, k}(t) & \sin \theta_{i, k}(t) & 0 \\
0 & 0 & 1
\end{array}\right], \\
& \Gamma_{2 i, k}(t)=1.6\left[\begin{array}{ccc}
\cos \theta_{i, k}(t) & \sin \theta_{i, k}(t) & 0 \\
0 & 0 & 1
\end{array}\right] .
\end{aligned}
$$

And the matrix norm condition in the theorem is $\max _{i=1,2 . N} \max _{t \in[0, \mathrm{~T}]} \rho_{1}\left(I-\delta_{i} \Gamma_{1}(t) B\left(\eta_{i, k}(t)\right)\right)=0.9998<1$.

The Fig. 6(a) and Fig. 6(b) show the formation effect of four WMRs at $k=5$ and $k=30$ when the channel noise $\boldsymbol{m}_{k}(t)=\boldsymbol{n}_{k}(t)=0$ and the sample period $\Delta T=0.001$, respectively. It can be seen that with the increase of learning times, the formation effect is more and more perfect. At the same time, we also show the control signal in the 30 iterations through Fig. ?.

To illustrate that the proposed control algorithm has better transient performance in the time domain, the position and angular errors generated by the controller in reference [17] are compared. As shown in Fig. 8(a) and Fig. 8(b), it is obvious that the transient performance of the non-learning controller is not ideal in the 
Figure 6

Plane formation trajectory of WMRs

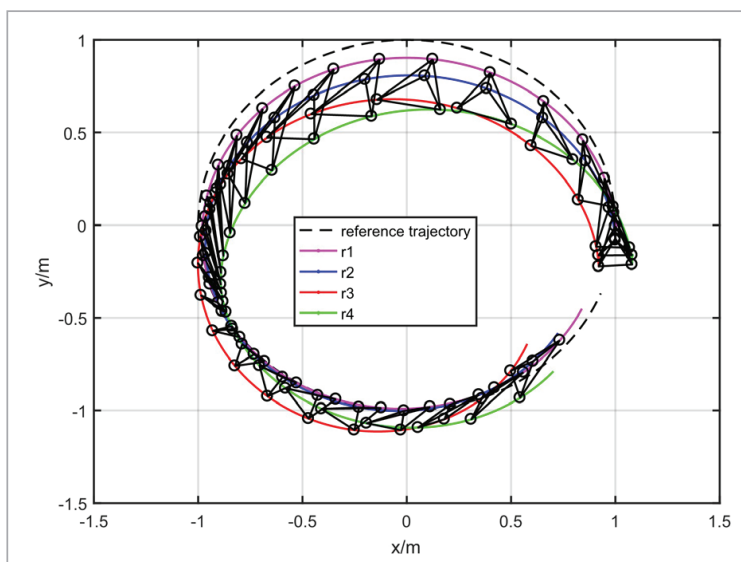

(a)

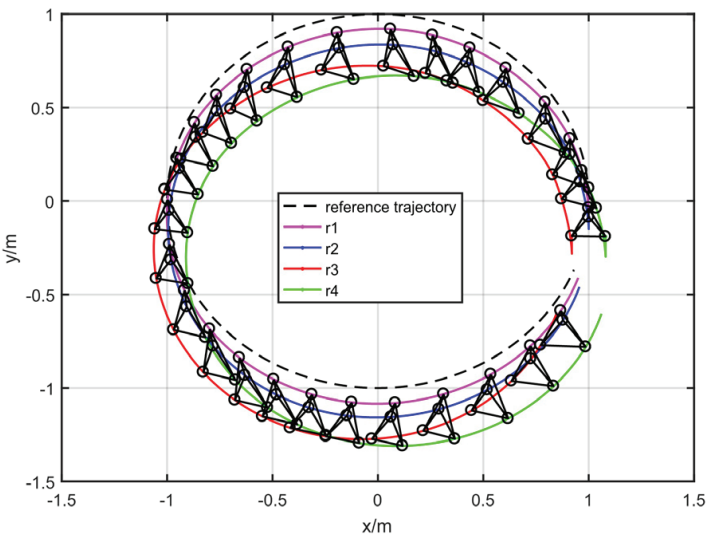

(b)

Figure 7

Control signals for WMRs
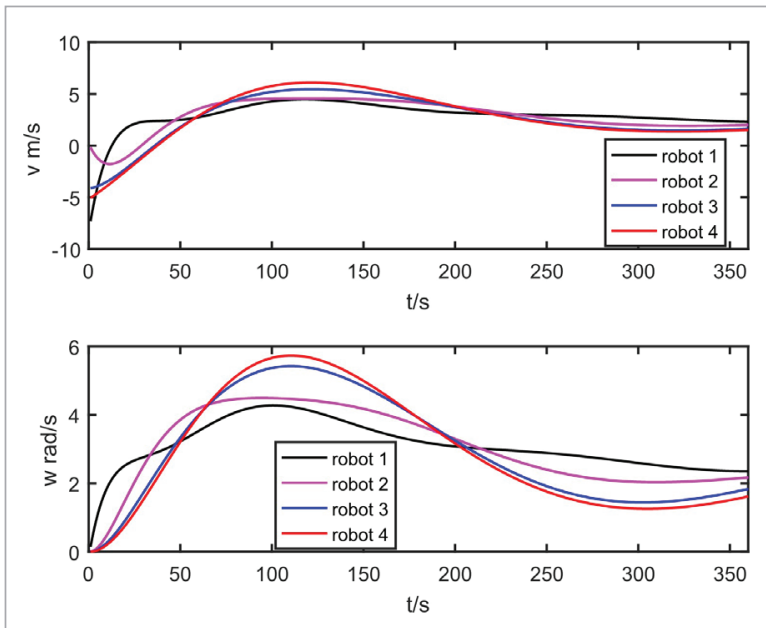

Figure 8

Comparison of transient performance between learning controller and non-learning controller

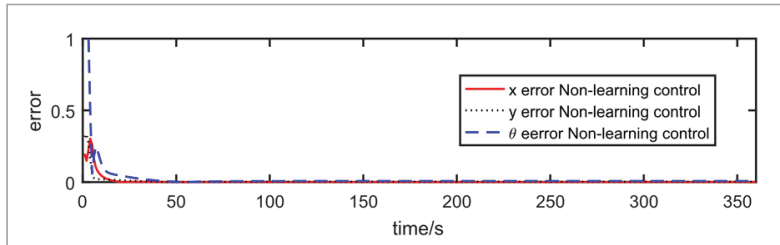

(a)

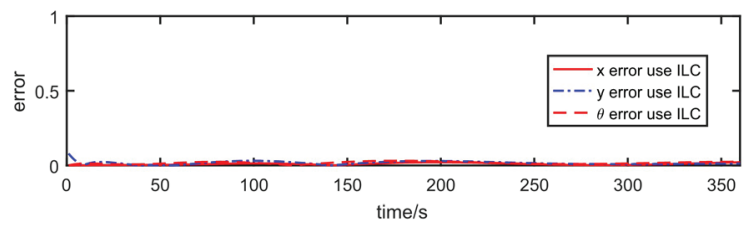

(b)

initial stage of control. By contrast, the learning controller can ensure the system has the ideal transient performance in the time domain after 30 iterations.

To illustrate the importance of the current learning item, a control algorithm (35) contains only a predicted learning item is adopted and the same simulation is performed again.

$$
\begin{aligned}
u_{i, k+1}(t) & =u_{i, k}(t)+\Gamma_{1}(t)\left\{\sum _ { j \in N _ { i } } a _ { i j } ( t ) \left(\delta \eta_{i, k}(t+1)\right.\right. \\
& \left.\left.-\delta \eta_{j, k}(t+1)\right)+s_{i}(t)\left(\delta \eta_{i, k}(t+1)\right)\right\} .
\end{aligned}
$$

Its controller learning gain is the same as (12).

The maximum position and angle errors of the multiple WMRs under the controller (12) and (35) are shown in Fig. 9. It can be observed that the two control algorithms are different in the convergence speed and performance. The convergence result under controller (12) over the 30 iterations shows faster convergence speed and better transient performance.

Next, we adjust different learning gains using controller (12) to compare their effects on control performance. The controller learning gain of the contrast group are selected as

$\Gamma_{1 i, k}(t)=0.8\left[\begin{array}{ccc}\cos \theta_{i, k}(t) & \sin \theta_{i, k}(t) & 0 \\ 0 & 0 & 1\end{array}\right]$,

$\Gamma_{2 i, k}(t)=0.8\left[\begin{array}{ccc}\cos \theta_{i, k}(t) & \sin \theta_{i, k}(t) & 0 \\ 0 & 0 & 1\end{array}\right]$. 


\section{Figure 9}

The influence of the current learning term on the maximum error of robot position and angle

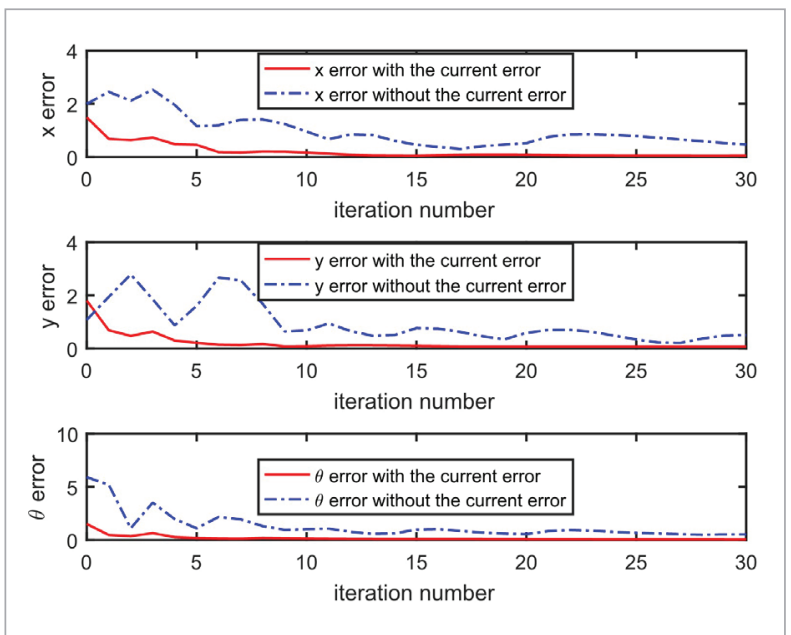

And the matrix norm condition in the theorem is $\max _{i=1,2 . . N} \max _{t \in[0, \mathrm{~T}]} \rho_{1}\left(I-\delta_{i} \Gamma_{1}(t) B\left(\eta_{i, k}(t)\right)\right)=0.9992<1$.

The original gain is denoted as $\Gamma$, and the gain of the contrast group is denoted as $\Gamma^{\prime}$.

The maximum position and angle errors of the multiple WMRs under different learning gains are shown in Fig. 10. It can be observed that different learning gains affect the transient performance of the system, but there is no difference in the final convergence effect.

\section{Figure 10}

The influence of different learning gains on the maximum error of robot position and heading

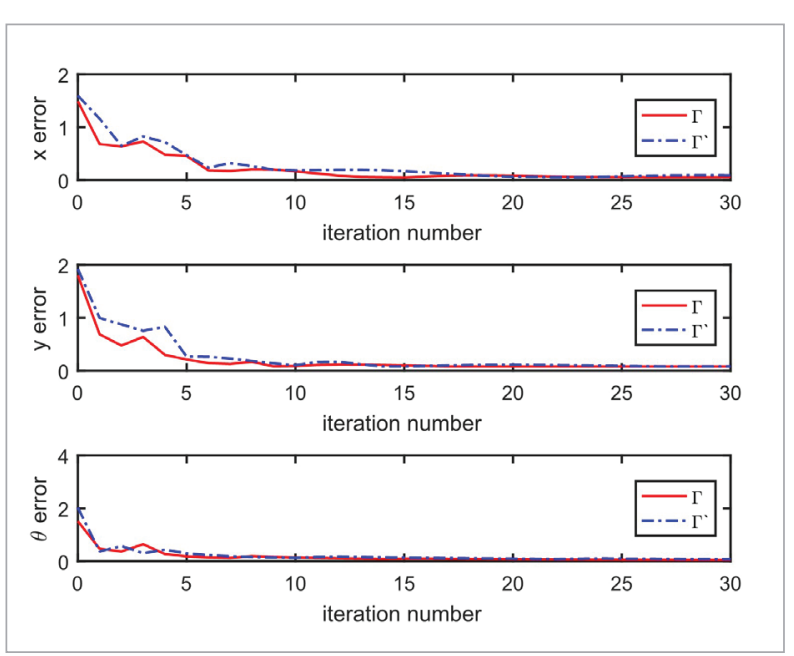

The tracking error convergence curve of the maximum position and angle of each WMR is shown in Fig. 11(a) - Fig. 11(d). Different sampling times were chosen for simulation, and the mean sum of CA and $\mathrm{SC}$ channel noises were 0.05 respectively. As can be seen from comparisons in these figures, the position and angle errors of WMR significantly decreased when a small sampling time was selected.

\section{Figure 11}

The convergence of position and angle errors for all WMRs with different sampling times

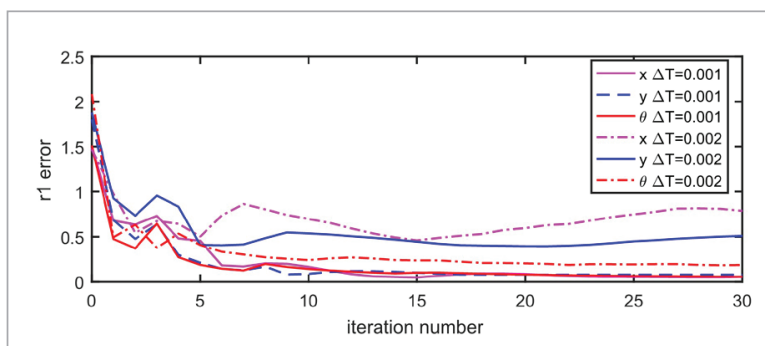

(a)

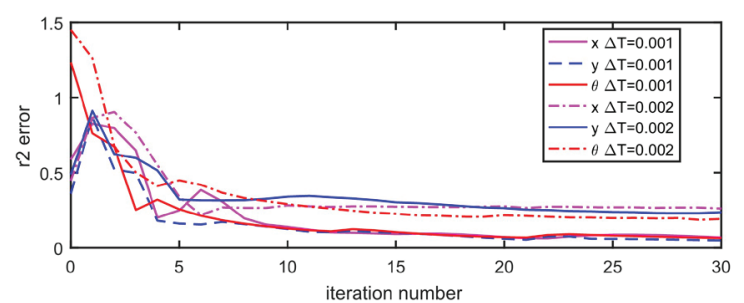

(b)

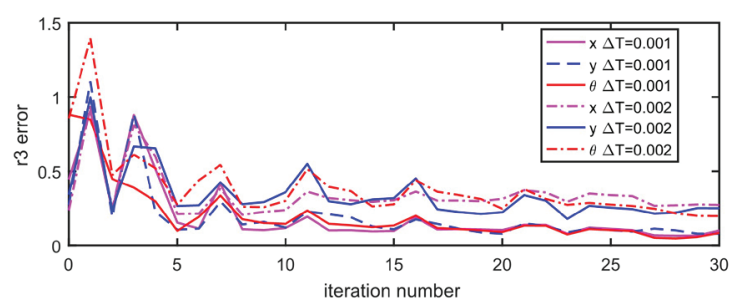

(c)

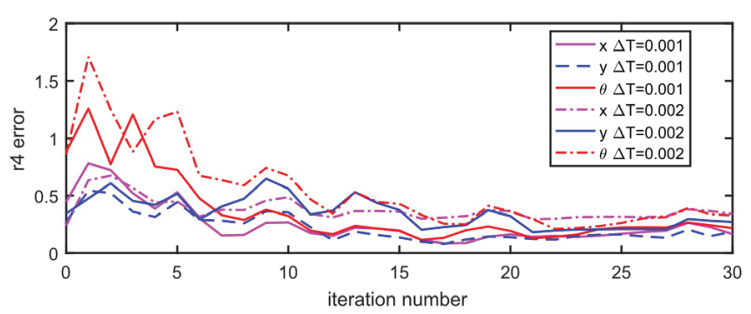

(d) 


\section{Conclusion}

In this paper, we studied the robust formation problem of multiple non-holonomic WMRs in repetitive motion environment, a distributed formation strategy based on ILC is proposed. The graph theory is introduced into the developed algorithm, which removes the condition that all WMRs need to obtain the desired trajectory information. The study used both predicted and current error as learning terms to improve the stability of the system under channel noise and switching topologies. The sufficient conditions for the stability are derived theoretically. Through analysis, it is found that the norm of system tracking error is related to the function of system control input matrix and channel noise, and the accumulation of CA and SC noise in the time domain and iteration domain can be suppressed by reducing the sampling time. The results show that the proposed algorithm

\section{References}

1. Antonelli, G., Arrichiello, F., Caccavale, F., Marino, A. Decentralized Time-Varying Formation Control for Multi-Robot Systems. The International Journal of Robotics Research, 2014, 33(7), 1029-1043. https://doi. org/10.1177/0278364913519149

2. Arrichiello, F., Chiaverini, S., Indiveri, G., Pedone, P. The Null-Space-based Behavioral Control for Mobile Robots with Velocity Actuator Saturations. International Journal of Robotics Research, 2010, 29(10), 1317-1337. https://doi.org/10.1177/0278364909358788

3. Bong, S., Park, J., Choi, Y. Adaptive Formation Control of Electrically Driven Nonholonomic Mobile Robots with Limited Information. IEEE Transactions on Systems Man and Cybernetics Part B, 2011, 41(4), 10611075. https://doi.org/10.1109/TSMCB.2011.2105475

4. Bu, X., Cui, Z., Cui, L., Qian, W. Robust Quantized H-Infinity ILC Design for Uncertain Systems with Communication Constraints. Information Technology and Control, 2018, 47(3), 564-574. https://doi.org/10.5755/ j01.itc. 47.3 .18451

5. Bu, X., Yu, F., Hou, Z., Yang, H. Robust Iterative Learning Control for Nonlinear Systems with Measurement Disturbances. Journal of Systems Engineering and Electronics, 2012, 23(6), 906-913. https://doi.org/10.1109/ JSEE.2012.00111 can realize the formation of multiple WMRs under channel noise and variable communication topology, and has better transient performance. At the same time, we observed that the number of times of learning is too much, which has a certain impact in practical engineering problems. This is also one of the questions that need further study in the future.

\section{Acknowledgement}

This work was supported by the National Natural Science Foundation of China (Nos. 61573129, 61573130, U1804147), the Innovative Scientists and Technicians Team of Henan Provincial High Education (20IRTSTHN019) and the Innovative Scientists and Technicians Team of Henan Polytechnic University (T2019-2).

6. Bu, X., Yu, Q., Hou, Z., Qian, W. Model Free Adaptive Iterative Learning Consensus Tracking Control for A Class of Nonlinear Multiagent Systems. IEEE Transactions on Systems, Man, and Cybernetics: Systems, 2019, 49(4), 677-686. https://doi.org/10.1109/ TSMC.2017.2734799

7. Freudenthaler, G., Meurer, T. PDE-based Multi-Agent Formation Control Using Flatness and Backstepping: Analysis, Design and Robot experiments. Automatica, 2020, 115, 108897. https://doi.org/10.1016/j.automatica.2020.108897

8. Huang,L.,Fang,Y.ConvergenceAnalysisofWirelessRemote Iterative Learning Control Systems with Dropout Compensation. Mathematical Problems in Engineering, 2013, 17(6), 2374-2381. https://doi.org/10.1155/2013/609284

9. Huang, L., Fang, Y., Wang, T. Method to Improve Convergence Performance of Iterative Learning Control Systems Over Wireless Networks in Presence of Channel Noise. IET Control Theory and Applications, 2014, 8(3), 175-182. https://doi.org/10.1049/iet-cta.2013.0399

10. Kecai, C., Jiang, B., Yue, D. Distributed Consensus of Multiple Nonholonomic Mobile Robots. IEEE/CAA Journal of Automatica Sinica, 2014, 1(2), 162-170. https://doi.org/10.1109/JAS.2014.7004546 
11. Khac, D., Lau, M. Practical Formation Control of Multiple Unicycle-Type Mobile Robots with Limited Sensing Ranges. Journal of Intelligent and Robotic Systems, 2011, 64(2), 245-275. https://doi.org/10.1007/s10846010-9531-7

12. Lan, Y., Wu, B., Shi, Y., Luo Y. Iterative Learning Based Consensus Control for Distributed Parameter Multi-Agent Systems with Time-Delay. Neurocomputing, 2019,357(10), r7-85. https://doi.org/10.1016/j.neucom.2019.04.064

13. Li, J., Li, J. Adaptive Iterative Learning Control for Consensus of Multi-Agent Systems. IET Control Theory and Applications, 2013, 7(1), 136-142. https://doi. org/10.1049/iet-cta.2012.0048

14. Liu, Y., Jia, Y. Formation Control of Discrete-Time Multi-Agent Systems by Iterative Learning Approach. International Journal of Control, Automation and Systems, 2012, 10(5), 913-919. https://doi.org/10.1007/ s12555-012-0507-1

15. Nguyen, T., Qiu, Z., Nguyen, T., Cao, M., Xie, L. Persistently Excited Adaptive Relative Localization and Time-Varying Formation of Robot Swarms. IEEE Transactions on Robotics, 2020, 36(2), 553-560.https:// doi.org/10.1109/TRO.2019.2954677

16. Ou, M., Du, H., Li, S. Finite-Time Tracking Control of Multiple Nonholonomic Mobile Robots. Journal of the Franklin Institute, 2012, 349(9), 2834-2860. https:// doi.org/10.1016/j.jfranklin.2012.08.009

17. Qian, D., Tong, S., Guo, J., Lee, S. Leader-Follower-based Formation Control of Nonholonomic Mobile Robots with Mismatched Uncertainties via Integral Sliding Mode. Proceedings of the Institution of $\mathrm{Me}-$ chanical Engineers, 2015, 229(I6), 559-569. https://doi. org/10.117r/0959651814568365

18. Qiu, Y., Xiang, L. Distributed Adaptive Coordinated Tracking for Coupled Nonholonomic Mobile Robots. IET Control Theory and Applications, 2014, 8(18), 2336-2345. https://doi.org/10.1049/iet-cta.2014.0099

19. Roza, A., Maggiore, M., Scardovi, L. A Smooth Distributed Feedback for Formation Control of Unicycles.
IEEE Transactions on Automatic Control, 2019, 64(12), 4998-5011. https://doi.org/10.1109/TAC.2019.2904152

20. Rozaria, R., Oomen, T. Data-Driven Iterative Inversion-based Control: Achieving Robustness Through Nonlinear Learning. Automatica, 2019, 107, 342-352. https://doi.org/10.1016/j.automatica.2019.05.062

21. Shao, J., Xie, G., Wang, L. Leader-Following Formation Control of Multiple Mobile Vehicles. IET Control Theory and Applications, 2007, 1(2), 545-552. https://doi. org/10.1049/iet-cta:20050371

22. Shi, J., Xu, J., Sun, J., Yang, Y. Iterative Learning Control for Time-Varying Systems Subject to Variable Pass Lengths: Application to Robot Manipulators. IEEE Transactions on Industrial Electronics, 2020, 67(10), 8629-837. https://doi.org/10.1109/TIE.2019.2947838

23. Sung, J., Kim, T. Predesignated Fault-Tolerant Formation Tracking Quality for Networked Uncertain Nonholonomic Mobile Robots in The Presence of Multiple Faults. Automatica, 2017, 77, 380-387. https://doi. org/10.1016/j.automatica.2016.09.011

24. Wang, Z., Wang, L., Zhang, H., Vlacic, L., Chen, Q. Distributed Formation Control of Nonholonomic Wheeled Mobile Robots Subject to Longitudinal Slippage Constraints. IEEE Transactions on Systems, Man, and Cybernetics: Systems, 2019, 99(1), 1-12.

25. Yang, S., Xu, J., Huang, D., Tan, Y. Optimal Iterative Learning Control Design for Multi-Agent Systems Consensus Tracking. Systems and Control Letters, 2014, 69, 80-89. https://doi.org/10.1016/j.sysconle.2014.04.009

26. Zhang, T., Li, J. Iterative Learning Control for Multiagent Systems with Finite-Leveled Sigma-Delta Quantization and Random Packet Losses. IEEE Transactions on Circuits and Systems I: Regular Papers, 2017, 64(8), 2171-2181. https://doi.org/10.1109/TCSI.2017.2690689

27. Zhao, Y., Zhang, F., Huang, P., Liu. X. Impulsive Super-Twisting Sliding Mode Control for Space Debris Capturing via Tethered Space Net Robot. IEEE Transactions on Industrial Electronics, 2020, 67(8), 68746882. https://doi.org/10.1109/TIE.2019.2940002 